\title{
Interactive comment on "Water-level attenuation in broad-scale assessments of exposure to coastal flooding: a sensitivity analysis" by Athanasios T. Vafeidis et al.
}

\section{Athanasios T. Vafeidis et al.}

vafeidis@geographie.uni-kiel.de

Received and published: 13 November 2017

We note the brief comment on our paper by a second anonymous reviewer. Based on the comments of both reviewers we realise that many readers may not be familiar with global coastal impact assessments. Therefore we have reworded and extended sections of the document to clarify those points that appear to have led to confusion. Our reply to the first reviewer does actually address the comments of the second reviewer. However we would also like to draw attention to the following important points, which we have also now explicitly addressed in our manuscript:

Printer-friendly version

Discussion paper

- Our paper does not simply "highlight the importance of accounting for the effects of 
water level attenuation" but actually quantifies the uncertainty related to these effects in global impact assessments. Importantly it shows that differences resulting from the use of different RCPs are of the same magnitude as differences resulting from the lack of accounting for water-level attenuation.

- As the reviewers mention, the need to account for such effects is well known to the community but not implemented due to computational constraints. We agree and would also like to add data constraints to those limitations, which are at least equally important and which we now discuss in detail in the manuscript.

- Our paper is the first study that addresses exactly these limitations for global coastal impact assessments under a full range of sea-level and socio-economic uncertainty. The study by Vousdoukas et al., (2016) is not global (contrary to what the reviewer suggests) and only assessed exposure for a limited set of physical scenarios (no socioeconomic) and a single event.

- The main reason why no other study to date has carried out a global impact assessment using a hydrodynamic model is, besides the input data volume, the large number of runs that one would need to conduct for the analysis. Using a hydrodynamic model in our study would have involved at least 60 runs (20 time steps for three physical scenarios), with the number increasing greatly when additional scenarios (including socio-economic ones) are considered. The reviewers might be unaware of the above data and processing limitations for impact assessments when suggesting the use of hydrodynamic models (implemented e.g. through the use of specific Manning values).

- We have conducted a sensitivity analysis (which is a form of validation), exactly because our study is global and involves future projections for a range of scenarios.

- Including land use information (that both reviewers suggest or imply) in our assessment (e.g. for deriving Manning values) is not possible as there are currently no global consistent land-use scenarios available. Contrary to what the reviewers suggest, (as we discussed in our response to the first reviewer), such data are not readily available.

Printer-friendly version

Discussion paper 
Even existing current global land-use data (not scenarios) suffer from severe limitations (e.g. resolution, relevant land use classes) when it comes to representing narrow coastal strips.

On a more general note, we would like to point out that the use of hydrodynamic models does not always guarantee improved results, as explicitly stated by Vousdoukas et al. (2016). The bathtub model has so far been the only option for global impact assessment as it can produce reasonable results for many locations and under specific conditions (as clearly stated in all the references cited by the first reviewer); and is representative of maximum potential impacts. Importantly, it is questionable whether a more detailed representation of the physical processes (in this case flooding) is the most urgent requirement of impact assessments as there are many sources of uncertainties (data, physical scenarios, socio-economic scenarios, damage estimations) that may as well be of equal importance. Most of the papers that have been cited by the reviewers support this argument by underlining the above uncertainties. Our work is a first step towards developing a simple model for assessing flooding at global scales, by quantifying one important source of uncertainty. Importantly it suggests the possibility of emulating physical processes through the application of simple correction factors and a typological approach.

Interactive comment on Nat. Hazards Earth Syst. Sci. Discuss., https://doi.org/10.5194/nhess2017-199, 2017.

Printer-friendly version

Discussion paper 